\title{
Chebysher Polynomials in the Numerical Solution of Differential Equations
}

\author{
By A. G. Morris and T. S. Horner
}

\begin{abstract}
Amongst satisfactory techniques for the numerical solution of differential equations, the use of Chebyshev series is often avoided because of the tedious nature of the calculations. A systematic application of the Chebyshev method is given for certain fourth order boundary value problems in which the derivatives have polynomial coefficients. Numerical results for various problems using the Chebyshev method are superior to those obtained by alternative methods.
\end{abstract}

1. Introduction. The solution of differential equations, including boundary value problems, with the solution expressed as a series of Chebyshev polynomials, is well known. See, for example, Clenshaw [1], and Fox and Parker [2]. The present paper is concerned with showing that some painstaking preliminary work can lead to information which can then be readily applied to obtain extremely accurate results using only a small number of terms in the Chebyshev series.

The problems to be considered are of the form

$$
\sum_{i=1}^{4} p_{i}(x, \lambda) \frac{d^{i} \phi}{d x^{i}}=0, \quad \phi( \pm 1)=\phi^{(1)}( \pm 1)=0
$$

where $\left\{p_{i}(x, \lambda)\right\}$ is a set of polynomials, quadratic in $x$, and linear in an eigenvalue $\lambda$.

In terms of notation to be used later

$$
\left\{\begin{array}{l}
p_{4}(x, \lambda)=c_{0}, \\
p_{3}(x, \lambda)=c_{1}+c_{2} x+c_{3} x^{2}, \\
p_{2}(x, \lambda)=c_{4}+c_{5} x+c_{6} x^{2}, \\
p_{1}(x, \lambda)=c_{7}+c_{8} x+c_{9} x^{2}, \\
p_{0}(x, \lambda)=c_{10}+c_{11} x+c_{12} x^{2}
\end{array}\right.
$$

where $c_{i}, i=0, \ldots, 12$, are linear functions of $\lambda$.

The general application of Chebyshev series to a simplified differential equation, where all $c_{i} \equiv 0$, except for $i=0,4,6$ is illustrated in Section 2. In Section 3, the problem of Eq. (1.1) is considered, and Section 4 contains numerical results obtained by using the Chebyshev method.

2. The Method of Solution. Assume that the solution of (1.1) can be written as a series of Chebyshev polynomials in the form

Received November 18,1975

AMS (MOS) subject classifications (1970). Primary 65L15; Secondary 65F15. 


$$
\begin{aligned}
\phi(x) & =\sum_{k=0}^{\infty}{ }^{\prime} a_{k} T_{k}(x) \\
& =1 / 2 a_{0}+a_{1} T_{1}(x)+a_{2} T_{2}(x)+\cdots,
\end{aligned}
$$

where $T_{k}(x)$ is the Chebyshev polynomial of the first kind, of degree $k$, given by

$$
T_{k}(x)=\cos \left(k \cos ^{-1} x\right)
$$

and $a_{k}$ is independent of $x$, but dependent (implicitly) on $\lambda$, and where $\Sigma^{\prime}$ indicates that the summation involves $1 / 2 a_{0}$ rather than $a_{0}$.

Similarly, the derivatives $\phi^{(i)}(x), i=0,1,2,3,4$, are written

$$
\phi^{(i)}(x)=\sum_{k=0}^{\infty} a_{k}^{(i)} T_{k}(x)
$$

where, by taking $a_{k}^{(0)} \equiv a_{k}$, this form includes the above expression for $\phi(x)\left(=\phi^{(0)}(x)\right)$.

Following [1] and [2], the standard recurrence relation

$$
2 x T_{k}(x)=T_{k+1}(x)+T_{|k-1|}(x)
$$

can be generalized to give

$$
(2 x)^{n} T_{k}(x)=\sum_{j=0}^{n}\left(\begin{array}{l}
n \\
j
\end{array}\right) T_{|k-n+2 j|}(x) .
$$

Then the fact that

leads to

$$
\begin{aligned}
\int T_{k}(x) d x & =1 / 2\left[\frac{T_{k+1}(x)}{k+1}-\frac{T_{|k-1|}(x)}{k-1}\right]+\text { constant, } \quad k \neq 1, \\
& =1 / 4 T_{2}(x)+\text { constant }, \quad k=1,
\end{aligned}
$$

$$
a_{k}^{(i)}-a_{k+2}^{(i)}=2(k+1) a_{k+1}^{(i-1)}, \quad k \geqslant 0,
$$

as a relation between the coefficients in the expansions of successive derivatives, after integrating term by term in

$$
\int \sum_{k=0}^{\infty} a_{k}^{(i)} T_{k}(x) d x=\sum_{k=0}^{\infty} a_{k}^{(i-1)} T_{k}(x)+\text { constant }
$$

and equating coefficients.

The general method of solution involves the substitution of the series (2.1) into (1.1) and then the use of (2.2) and (2.3) to obtain a set of results of the form

$$
\sum_{l=k-m}^{k+m}\left\{\sum_{j=0}^{12} c_{j} w_{l j}\right\} a_{l}=0, \quad k=s, s+r, s+2 r, \ldots,
$$

after equating coefficients of $T_{k}(x)$.

The idea can be illustrated with respect to the simple fourth order equation 


$$
c_{0} \phi^{(4)}(x)+\left(c_{4}+c_{6} x^{2}\right) \phi^{(2)}(x)=0
$$

On equating coefficients of $T_{k}(x)$, after substituting (2.1) into (2.5), and using (2.2), the result

$$
c_{0} a_{k}^{(4)}+c_{4} a_{k}^{(2)}+1 / 4 c_{6}\left[a_{k-2}^{(2)}+2 a_{k}^{(2)}+a_{k+2}^{(2)}\right]=0, \quad k \geqslant 0,
$$

is obtained.

Rewriting this equation with $k$ replaced firstly by $k-1$, and then by $k+1$, and subtracting one of the resulting expressions from the other, gives

$$
2 c_{0} k a_{k}^{(3)}+2 c_{4} k a_{k}^{(1)}+1 / 4 c_{6}\left[2(k-2) a_{k-2}^{(1)}+4 k a_{k}^{(1)}+2(k+2) a_{k+2}^{(1)}\right]=0, \quad k \geqslant 1 .
$$

By repeating the procedure on the above relation, and also on two subsequent relations, eventually a relation is obtained containing only the $a_{k}\left(=a_{k}^{(0)}\right)$, i.e.,

$$
\begin{aligned}
\left\{\frac{c_{6}(k-5)(k-4)}{16(k-3)(k-2)(k-1) k}\right\} a_{k-4} \\
+\left\{\frac{c_{4}}{4(k-1) k}+\frac{c_{6}(5 k-19)}{8(k-3)(k-1) k(k+1)}\right\} a_{k-2} \\
+\left\{c_{0}-\frac{c_{4}}{2(k-1)(k+1)}-\frac{c_{6}\left(k^{2}-22\right)}{8(k-2)(k-1)(k+1)(k+2)}\right\} a_{k} \\
+\left\{\frac{c_{4}}{4 k(k+1)}-\frac{c_{6}(5 k+19)}{8(k-1) k(k+1)(k+3)}\right\} a_{k+2} \\
+\left\{\frac{c_{6}(k+4)(k+5)}{16 k(k+1)(k+2)(k+3)}\right\} a_{k+4}=0, \quad k \geqslant 4 .
\end{aligned}
$$

(2.6) is in the same form as (2.4).

It can now be more readily seen that each of the bracketed terms is an inner product of the vector $\mathrm{c}=\left[c_{0}, c_{1}, \ldots, c_{12}\right]$ and a vector $\mathrm{w}_{l}$ whose components are a function of $k$. Table 1 exhibits a matrix $W$, in which each row is one of these vectors $\mathrm{w}_{l}, l=k-6, \ldots, k+6$.

In the outer summation of Eq. (2.4), the value of $m$ can always be taken as 6 , but because of the number of zero elements in $W$, the value of $m$ may often be adjusted to a smaller value. For example, $m=4$ in Eq. (2.6). The outer summation often proceeds in steps of 2, and allowance for the occurrence of steps of 2 can also be made in a computer program using (2.4).

Similarly, the inner summation with respect to $j$ can be modified to involve steps of 2 , because of the alternate zero elements in $\mathbf{w}_{l}$, and there are often further simplifications because $c_{j}=0$ for some values of $j$. 


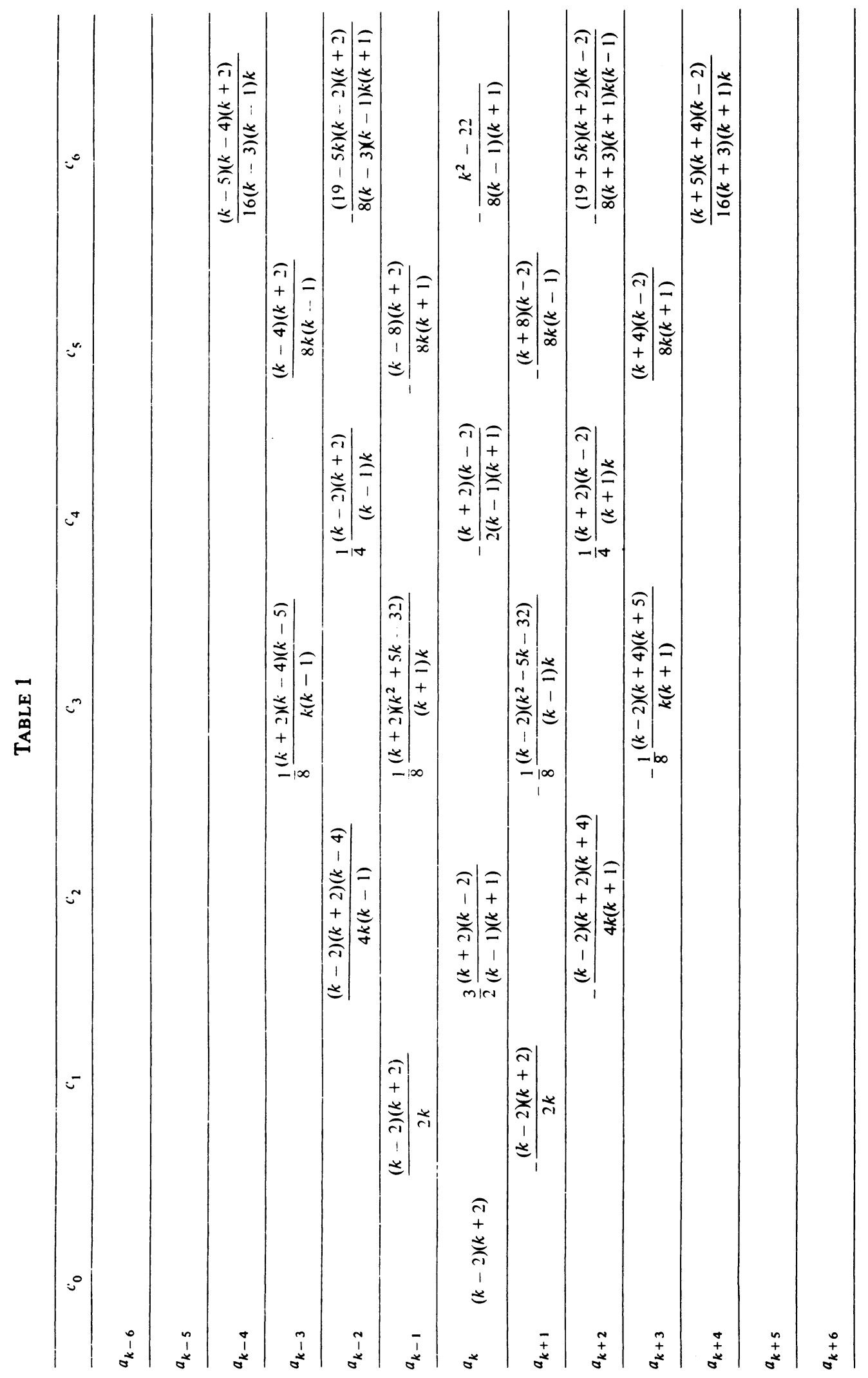




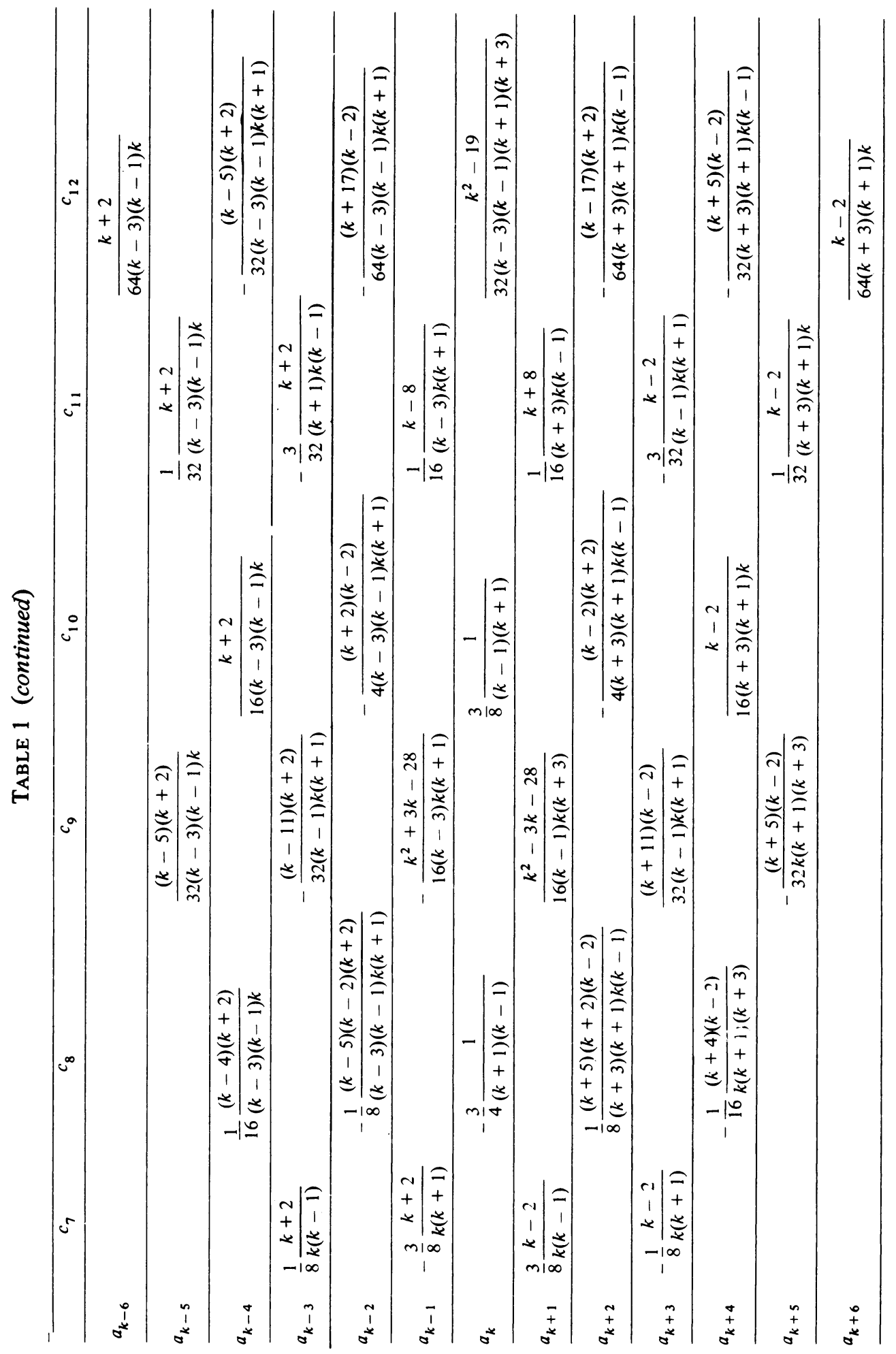


The number of results of the type (2.4) is equal to $n$, the order of the matrix to be used in the eigenvalue problem. The initial value of $k$ is given by $s=4,4$ or 3 , respectively, depending on whether the problem involves a general, even or odd solution, as will be explained in Section 3. Similarly, the increment in $k$ is given by $r=$ $1,2,2$, respectively.

3. Boundary Value Problem. When substituted into (2.1), the boundary value conditions, $\phi( \pm 1)=\phi^{(1)}( \pm 1)=0$, lead to

$$
\begin{array}{r}
1 / 2 a_{0}+a_{2}+a_{4}+\cdots=0, \\
a_{1}+a_{3}+a_{5}+\cdots=0, \\
1 / 2 a_{0}^{(1)}+a_{2}^{(1)}+a_{4}^{(1)}+\cdots=0, \\
a_{1}^{(1)}+a_{3}^{(1)}+a_{5}^{(1)}+\cdots=0 .
\end{array}
$$

Use of (2.3) in (3.1c) and (3.1d) give the equivalent results

$$
\begin{aligned}
& 1^{2} \cdot a_{1}+3^{2} \cdot a_{3}+5^{2} \cdot a_{5}+\cdots=0 \\
& 2^{2} \cdot a_{2}+4^{2} \cdot a_{4}+6^{2} \cdot a_{6}+\cdots=0
\end{aligned}
$$

These results finally lead to

$$
\begin{aligned}
1 / 2 a_{0} & =-\sum_{k=1}^{\infty} a_{2 k}, \\
a_{2} & =-\sum_{k=2}^{\infty} k^{2} a_{2 k}, \\
a_{1} & =\frac{1}{2} \sum_{k=2}^{\infty}(k+2)(k-1) a_{2 k+1}, \\
a_{3} & =-\frac{1}{2} \sum_{k=2}^{\infty} k(k+1) a_{2 k+1},
\end{aligned}
$$

so that, in conjunction with the fact that $a_{-k}=a_{k}$, the equations of type (2.4) can be modified to start with terms involving $a_{4}, a_{5}, \ldots$

An even solution to (1.1) can be written

$$
\phi(x)=\sum_{k=0}^{\infty} a_{2 k} T_{2 k}(x)
$$

since

$$
T_{r}(-x)=(-1)^{r} T_{r}(x)
$$

and the boundary conditions become (3.2a) and (3.2b) only.

An odd solution can be written 


$$
\phi(x)=\sum_{k=0}^{\infty} a_{2 k+1} T_{2 k+1}(x)
$$

with boundary conditions (3.2c) and (3.2d).

Of course, most problems will not have specialized solutions of even or odd type, and then the general set of boundary conditions (3.2) must be used.

Now, the coefficients $c_{i}$ in (1.1) are linear functions of an eigenvalue $\lambda$, so that with $c_{i}=\alpha_{i}-\lambda \beta_{i}$ (say), and with the use of Table 1 and the boundary conditions (3.2), the eigenvalue problem reduces to the generalized algebraic problem

$$
[A-\lambda B] \mathbf{a}=\mathbf{0},
$$

where

$$
\mathbf{a}^{T}= \begin{cases}{\left[a_{4}, a_{5}, a_{6}, \ldots, a_{N}\right] \text { for the general problem, }} \\ {\left[a_{4}, a_{6}, \ldots, a_{N}\right] \text { for the even problem, }} \\ {\left[a_{3}, a_{5}, \ldots, a_{N}\right] \text { for the odd problem, }}\end{cases}
$$

and where $N$ is sufficiently large for suitable accuracy, and is the cutoff point in the Chebyshev expansion, after which the terms are assumed to be zero.

4. Numerical Results. The algebraic problem was solved directly by considering

$$
[D-\lambda I] \mathbf{a}=\mathbf{0},
$$

where $D=B^{-1} A$.

The QR algorithm of Francis [3] was applied to a Hessenberg matrix similar to $D$. The form of the algorithm was as described by Parlett [6]. At present, the methods of Moler and Stewart [5], and Kaufman [4] are being investigated as alternatives for solving the generalized algebraic problem.

The preceding analysis was used on a number of fourth order differential equations, including a number of problems in fluid mechanics. For certain simple examples, the following results were obtained. All calculations were performed on the UNIVAC 1106 (with $1.5 \mu \mathrm{sec}$ memory) installation at the University of Wollongong, N.S.W., Australia.

(i) $\phi^{(4)}+\lambda \phi^{(2)}=0, \phi(1)=\phi^{(1)}(1)=0$, Even Solution. The exact eigenvalues of this equation for the even solution are $\lambda=m^{2} \pi^{2}, m=1,2, \ldots$

The smallest eigenvalue, for various values of $m$ (size of matrix), is given below:

$\begin{array}{cc}m & \lambda \\ 4 & 9.8698208 \\ 5 & 9.8696003 \\ 6 & 9.8696045 \\ 7 & 9.8696044\end{array}$

The exact value to eight significant figures is 9.8696044 .

For $m=7$, a comparison is given for the first four eigenvalues. 
Eigenvalue number

1
2
3
4 $\lambda$

9.8696044
39.478414
88.832161

Exact

9.8696044

39.478417

88.826439

157.91367

For $m=12$, the first seven figures are exact for eigenvalues 2, 3 and 4. For $m=7$, the CPU time for all seven eigenvalues was 0.9 secs.

When the eigenvectors are found as well, as the eigenvalues, then the actual series representing the eigenfunction can be found. Further, the compact evaluation scheme for Chebyshev polynomials, viz.,

$$
\left\{\begin{array}{l}
b_{n+2}=b_{n+1}=0, \\
b_{r}=2 x b_{r+1}-b_{r+2}+a_{r}, \\
\phi(x)=1 / 2\left(b_{0}-b_{2}\right),
\end{array}\right.
$$

can be used to evaluate the eigenfunction $\phi(x)$ for required values of $x$.

In the present example, the eigenfunction $\phi(x)$ is given by

$$
\phi(x) \doteqdot \sum_{k=0}^{9} a_{2 k} T_{2 k}(x),
$$

where the coefficients are given for the eigenvalues $\lambda=9.8696044$ and $\lambda=39.478414$ in Table 2. In Table 3 the tabulated values of $\phi(x)$ are given for $x=0(0.1) 1.0$. The eigenfunctions are known to be $1 / 2(1+\cos \pi x)$ and $1 / 2(1-\cos 2 \pi x)$, giving values in exact agreement with those in Table 3.

(ii) $\phi^{(4)}+\lambda \phi^{(2)}=0, \phi(1)=\phi^{(1)}(1)=0$, Odd Solution. The exact eigenvalues are given by $\lambda=\alpha^{2}$, where $\alpha=\tan \alpha$, and the present method gives the first two eigenvalues as 20.190729 and 59.679516 , exact to the given number of figures, using a matrix of order 10 . The associated eigenfunctions are given in Table 4 . They have been normalized so that $a_{1}=1$.

(iii) $\phi^{(4)}-\lambda \phi=0, \phi(1)=\phi^{(1)}(1)=0$, Even Solution. The exact eigenvalues of this equation for the even solution are $\lambda=\alpha^{4}$ where $\tan \alpha=-\tanh \alpha$.

The smallest eigenvalue, for various values of $m$ (size of matrix), is given below:

$$
\begin{array}{cc}
m & \lambda \\
4 & 31.285316 \\
5 & 31.285243
\end{array}
$$

The exact eigenvalue to eight significant figures is 31.285243 .

For $m=12$, the first seven figures are exact for the first four eigenvalues.

For $m=5$, the CPU time for the calculation of all eigenvalues was 0.5 secs.

(iv) $\phi^{(4)}-\lambda \phi=0, \phi(1)=\phi^{(1)}(1)=0$, Odd Solution. The exact eigenvalues are given by $\lambda=\alpha^{4}$ where $\tan \alpha=\tanh \alpha$, and the present method gave the first two eigenvalues as 237.72106 and 2496.4874 , exact to the given number of figures using a matrix of order 10 . 
TABLE 2

Coefficients in the expansion $\phi(x)=\Sigma_{k=0}^{\prime \infty} a_{2 k} T_{2 k}(y)$ for the problem in Section 4(i)

\begin{tabular}{lrr}
\hline & $\lambda=9.8696044$ & $\lambda=39.47842$ \\
\hline $1 / 2 a_{0}$ & 0.34787891 & 0.38986155 \\
$a_{2}$ & -0.48543393 & -0.28788036 \\
$a_{4}$ & 0.15142457 & -0.31568048 \\
$a_{6}$ & -0.01454597 & 0.27768840 \\
$a_{8}$ & 0.00069612 & -0.07329532 \\
$a_{10}$ & -0.00002010 & 0.01013846 \\
$a_{12}$ & 0.00000039 & -0.00088337 \\
$a_{14}$ & & 0.00005342 \\
$a_{16}$ & & -0.00000238 \\
$a_{18}$ & & 0.00000008 \\
\hline
\end{tabular}

TABLE 3

Values of $\phi(x)$ for $0 \leqslant x \leqslant 1$, for the problem in Section 4(i)

\begin{tabular}{ccc}
\hline$x$ & $\lambda=9.8696044$ & $\lambda=39.47842$ \\
\hline 0.0 & 1.000000 & 0.000000 \\
0.1 & 0.975528 & 0.095492 \\
0.2 & 0.904508 & 0.345492 \\
0.3 & 0.793893 & 0.654509 \\
0.4 & 0.654508 & 0.904509 \\
0.5 & 0.500000 & 1.000000 \\
0.6 & 0.345492 & 0.904509 \\
0.7 & 0.206107 & 0.654509 \\
0.8 & 0.095492 & 0.345492 \\
0.9 & 0.024472 & 0.095492 \\
1.0 & 0.000000 & 0.000000 \\
\hline
\end{tabular}


TABLE 4

Coefficients in the expansion $\phi(x)=\Sigma_{k=0}^{\infty} a_{2 k+1} T_{2 k+1}(x)$ for the problem in Section 4(ii)

\begin{tabular}{lrr}
\hline & $\lambda=20.190729$ & $\lambda=59.67952$ \\
\hline$a_{1}$ & 1.00000000 & 1.00000000 \\
$a_{3}$ & -1.64282456 & -0.90727902 \\
$a_{5}$ & 0.74907228 & -0.78563334 \\
$a_{7}$ & -0.11505988 & 0.97653959 \\
$a_{9}$ & 0.00926122 & -0.33922204 \\
$a_{11}$ & -0.00046468 & 0.06233792 \\
$a_{13}$ & 0.00001601 & -0.00730968 \\
$a_{15}$ & -0.00000040 & 0.00060179 \\
$a_{17}$ & & -0.00003692 \\
$a_{19}$ & & 0.00000176 \\
$a_{21}$ & & 0.00000007 \\
\hline
\end{tabular}

\section{Conclusion.}

(i) Comparison With Finite Difference Techniques. Results using the Chebyshev method were compared with those obtained using finite difference methods described by Osborne [6]. In all cases, the present method proved far superior.

For example, in the problem described above in Section 4(iii), the finite difference method gave approximations of $31.286328,31.285565$ and 31.285339 for matrices of order 12,18 and 27 respectively, compared with the exact value of 31.285243 which was obtained by the Chebyshev method using a matrix of order 5 .

The order of the matrices was chosen $(12=2 / 3 \cdot 18,18=2 / 3 \cdot 27)$ so that it would be useful if it was decided that $h^{p}$-extrapolation was appropriate, and in fact the choice of $p=4$ seemed correct. The improved results with this choice were 31.28538 and 31.28529.

(ii) General. The only major disadvantage of the Chebyshev technique in a practical situation is the tedious work in calculating the matrix $W$. Once $W$ has been found, the solution of the remainder of the problem is very routine, and the results obtained are excellent.

A further advantage (distinct from accuracy) of the Chebyshev method over the finite difference technique is that the eigenvector calculation is only done once for all values of $x$, when the eigenfunctions are sought. The value of $x$ is not introduced until calculations using system (4.1) are performed. For the finite difference method, 
the matrices $A$ and $B$, when calculated, depend on the values at the equidistant sample points; and if eigenfunction values are desired for other values of $x$, an interpolation is required.

The Chebyshev technique can easily be extended to complex valued boundary value problems of the same class, except that a modified $Q R$ algorithm may be required for solving the algebraic problem.

Department of Mathematics

University of Wollongong

Wollongong, NS.W., Australia

1. C. W. CLENSHAW, "The numerical solution of linear differential equations in Chebyshev series," Proc. Cambridge Philos. Soc., v. 53, 1957, pp. 134-149. MR 18, 516.

2. L. FOX \& I. B. PARKER, Chebyshev Polynomials in Numerical Analysis, Oxford Univ. Press, London and New York, 1968. MR 37 \#3733.

3. J. G. F. FRANCIS, "The $Q R$ transformation: A unitary analogue to the $L R$ transformation." I, II, Comput. J., v. 4, 1961/62, pp. 265-271, 332-345. MR 23 \#B3143; 25 \#744.

4. L. KAUFMAN, The LZ Algorithm to Solve the Generalized Eigenvalue Problem, Report STAN-CS-73-363, Computer Science Department, Stanford University, Stanford, California, 1973.

5. C. B. MOLER \& G. W. STEWART, "An algorithm for the generalized matrix eigenvalue problem $A x=\lambda B x$," SIAM J. Numer. Anal., v. 10, 1973, pp. 241-256. MR 49 \#10135.

6. M. R. OSBORNE, "Numerical methods for hydrodynamic stability problems," SIAM $J$. Appl. Math., v. 15, 1967, pp. 539-557. MR 39 \#5066.

7. B. N. PARLETT, "The $L U$ and $Q R$ algorithms," an extract from Mathematical Methods for Digital Computers. Vol. II, A. Ralston \& H. S. Wilf, Editors, Wiley, New York, 1967. MR 35 \#2516. 